\title{
A Hybrid $\mathrm{H}_{\infty}$ Control Based ILC Design Approach for Trajectory Tracking of a Twin Rotor Aerodynamic System
}

\author{
Faisal Saleem $^{1 \mathrm{a}}$, Ahsan Ali ${ }^{1 \mathrm{~b}}$, Inam ul Hasan Shaikh ${ }^{1 \mathrm{c}}$, Muhammad Wasim ${ }^{1 \mathrm{~d}}$
}

RECEIVED ON 21.02.2019, ACCEPTED ON 26.07.2019

\begin{abstract}
This paper investigates the trajectory tracking problem for a Multi-Input Multi-Output (MIMO) Twin Rotor Aerodynamic System (TRAS) using a hybrid architecture based on an $\mathbf{H}_{\infty}$ controller and Iterative Learning Control (ILC). TRAS is a fast, nonlinear coupled system and therefore it is a challenging task to design a control system that ensures the tracking for fast changing trajectories. The controllers proposed in the literature for the TRAS through linear approaches tend to have a large control effort, while the ones designed using the nonlinear approaches track only for smooth input trajectories. Both issues are important from control point of view. In this paper, these issues are addressed by designing a feedback $H_{\infty}$ control that stabilizes the system and a feedforward ILC which reduces the control effort. The $\mathrm{H}_{\infty}$ controller achieves the tracking for input trajectories with sharp edges, but the control effort required for tracking is large. With the proposed hybrid approach, tracking is achieved by the $\mathrm{H}_{\infty}$ controller whereas the required control effort is reduced in each subsequent iteration by ILC. After a few iterations, accurate tracking at a minimized control effort is achieved. The simulations have been performed using MATLAB software and the controller designed through the proposed approach has been validated on nonlinear model of the system. The results of the proposed technique, compared with the flatness-based and back-stepping control strategies, show that the proposed controller ensures accurate tracking at the reduced control effort.
\end{abstract}

Keywords: Twin Rotor Multi-Input-Multi-Output System, Iterative Learning Control, Flatness-Based Controller, Backstepping Controller.

\section{INTRODUCTION}

$\mathrm{T}$ he Unmanned Arial Vehicles (UAV) like fixed wing crafts, quadcopters, and helicopters have found applications in several domains [1]. Amongst these, quadcopters and helicopters are commonly used as UAVs due to their hovering ability. These systems possess nonlinear and coupled dynamics, which leads to the challenges in their autonomous control. The dynamics of helicopter can be approximated by a laboratory setup namely TRAS [2]. Like helicopter, TRAS has two rotors namely main and tail rotors. Though in helicopter, main rotor is able to tilt in order to execute forward motion [3].

TRAS is a MIMO system with a beam, able to freely execute motion both in vertical (pitch) and horizontal (azimuth) planes about a fixed point. The motion of beam is damped by counterbalance weights rigidly fixed at its center. Beam is equipped by two propellers mounted on its both ends. There is no pitch angle associated with propellers and thus corresponding forces solely depend on their rotational speeds of the propellers [2].

\footnotetext{
${ }^{1}$ Department of Electrical Engineering, University of Engineering and Technology, Taxila, Pakistan.

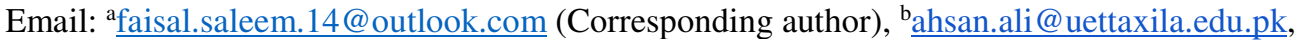
cinam.hassan@uettaxila.edu.pk, ${ }^{\mathrm{d}}$ muhammad.077wasim@gmail.com

This is an open access article published by Mehran University of Engineering and Technology, Jamshoro under CC BY 4.0 International License.
} 
In autonomous applications, trajectory tracking is one of the most basic and important tasks. Other equally important scenarios involve e.g., hover control. In [4], four independent Proportional Integral Derivative (PID) controllers with independent inputs for control have been designed to achieve the objective of trajectory tracking. Here, real value type genetic algorithm has been used to tune the controller parameters in order to reduce total error and control efficiency. System performance index is used as a fitness function here. A hybrid flight control system based on traditional PID and fuzzy control approaches for a single rotor simulated Raptor-30 V2 helicopter is presented in [5]. In [6], vibration suspension and motion control of twin rotor has been addressed by designing a GA-tuned PID controller. In [7], robust PID based dead beat control scheme is proposed. Although the model based PID controller can be designed for the system of interest, but the decoupling is required to transform a MIMO system into several SISO (Single-Input and Single-Output) systems which requires an extra computational effort. Moreover, this introduces problems from input uncertainty point of view [8]. To counter these problems, different MIMO model-based controllers have been reported in the literature. In [9] a Linear Quadratic Regulator (LQR) controller is designed. In [10], an LQR controller with integral action is applied to TRAS. In [11], mixed sensitivity robust $H_{\infty}$ controller has been designed for trajectory tracking of a twin rotor. In [12], a Model Predictive Controller (MPC) is designed for trajectory tracking of TRAS. As MPC solves the optimization problem online at each iteration, this solution is computationally expensive.

Nonlinear control approaches based on the nonlinear model of the system have also been reported. Different variants of Sliding Mode Controller (SMC) are proposed in [13], variants of Back Stepping Controllers (BSC) in [14] and Flatness Based Controller (FBC) in [15]. In [16], feedback linearization is used to design a nonlinear controller for TRAS. The quasi- Linear Parameter Varying (LPV) control is used in [17] where TRAS model has been first transformed into discrete time polytopic quasi-LPV model. In [18], an SMC is designed for the trajectory tracking problem of TRAS. As SMC controller faces chattering phenomena for control inputs so its different variants have been proposed. In [19], a fuzzy sliding and fuzzy integral sliding mode controllers are proposed for positioning the pitch and yaw angles. In [20], the tuning mechanism for Fuzzy SMC using PSO (Particle Swarm Optimization) algorithm is introduced. In order to alleviate chattering, twisting algorithm along with SMC is proposed in [21]. In order to nullify the effect of model uncertainties and external disturbances, the adaptive SMC with twisting algorithm is used in [22].

In [23], the disturbance observer based integral BSC is proposed for the trajectory tracking problem of TRAS. However, virtual BSC derivatives using command filter have been calculated which degrade the transient performance. In [24], dual boundary conditional integral BSC is designed for TRAS. Here, virtual BSC derivatives are calculated using analytical methods and the method is able to asymptotically regulate constant and time varying reference signals without degrading the transient response. It is concluded that both control approaches robustly track the desired signal but backstepping based SMC lumped with Extended Kalman Filter (EKF) observer has improved performance in both the transient and steady state cases. In [25], flatness based nonlinear control method is proposed for the trajectory tracking problem.

Nonlinear methods mentioned above require that the desired trajectory be twice differentiable [26]. As a consequence, if desired trajectory has sharp edges, then its first and second derivatives result in a large value and the closed-loop system loses stability. Backstepping, SMC and Flatness based controllers ensure closed-loop asymptotic stability for smooth differentiable trajectory. However, the control effort calculated using these nonlinear control design approaches, even for the smooth edges can be quite high.

Motivated by the issue mentioned above, a hybrid design approach based on ILC has been proposed in this paper to address both the control effort and trajectory tracking problems for TRAS. That is, the issue of trajectories with sharp edges is addressed in 
this work. A similar hybrid ILC control approach is proposed in [27] with Fractional Order PID controller in the inner loop for system stabilization. This hybrid approach was applied on a SISO system. However, in this paper the hybrid ILC strategy is improved by applying $\mathrm{H}_{\infty}$ controller in the inner loop which leads to lesser number of iterations for ILC. Moreover, the system selected is MIMO and contains coupling in the channels making control design task difficult in this case. The main contributions of the paper include:

(1) Improvement of already proposed hybrid ILC design approach with $\mathrm{H}_{\infty}$ controller in the inner loop for system stabilization, leading to the lesser number of iterations required to achieve the tracking compared to that proposed in [27].

(2) Application and validation of hybrid ILC control design approach on a coupled, nonlinear, MIMO system.

(3) Solution of the trajectory tracking for TRAS with reference trajectories having sharp edges.

Rest of the paper is organized as follows: section 2 describes the control oriented mathematical modeling of TRAS. Section 3 briefly discusses the control design approach proposed in the paper. Discussion on the simulation results is presented in section 4 and finally, conclusions are drawn in section 5 .

\section{MATHEMATICAL MODELING OF THE TRAS}

TRAS has a beam and two propellers which are named as the main propeller and the tail propeller. The propellers are connected at the ends of beam and DC motors are used to operate them [15].

The beam is pivoted and is able to move freely in both vertical and horizontal axes. The counterbalance weights for determining the pitch angle with no propeller action, are fixed at the end of the beam. The restriction of movement of the beam in terms of its pitch $(\theta)$ and azimuth $(\psi)$ angles is given as follows [15]:

$-3.2 \mathrm{rad}<\psi<3.2 \mathrm{rad}, \quad-1 \mathrm{rad}<\theta<1 \mathrm{rad}$
Inertial reference frame of the TRAS is shown in Fig. 1.

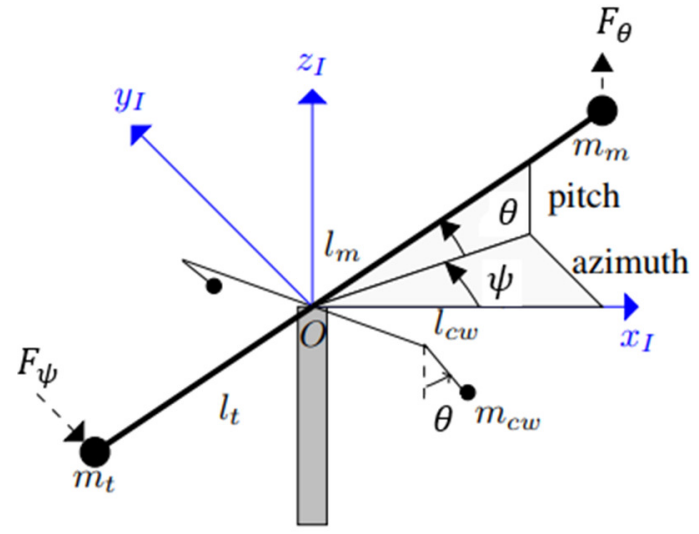

Fig. 1: Inertial Reference Frame of TRAS [14]

For the purposes of mathematical modelling, a righthanded coordinate system is located at the pivot point of beam. Origin of the coordinate system is denoted by 0 . The distance of tail and main rotors from the origin is denoted by $l_{t}$ and $l_{m}$ respectively. Whereas $m_{m}$ and $\mathrm{m}_{\mathrm{t}}$ represent the counterweights at the main and tail of the beam. Moreover, $m_{c \omega}$ and $l_{c \omega}$ represent the masses at the ends of levers and the relevant lengths of the levers respectively. The nonlinear model of the TRAS is given by the Equation (1):

$\mathrm{J}_{\psi} \ddot{\psi}=\mathrm{u}_{\psi}+\mathrm{J} \dot{\psi} \dot{\theta} \sin (2 \theta)-\mathrm{K}_{\psi} \psi-\mathrm{C}_{\psi} \dot{\psi}$,

$\mathrm{J}_{\theta} \ddot{\theta}=\mathrm{u}_{\theta}-\mathrm{J} \frac{\dot{\psi}^{2}}{2} \sin (2 \theta)-\mathrm{C}_{\theta} \dot{\theta}-\mathrm{K}_{\mathrm{g}} \mathrm{g}$

where,

$J_{\Psi}=\left(m_{m} l_{m}^{2}+m_{t} l_{t}^{2}\right) \cos ^{2}(\theta)+2 m_{c \omega} l_{c \omega} \sin ^{2}(\theta)+$ $\mathrm{J}_{\mathrm{z}}$

$\mathrm{J}_{\theta}=\mathrm{m}_{\mathrm{m}} \mathrm{l}_{\mathrm{m}}^{2}+\mathrm{m}_{\mathrm{t}} \mathrm{l}_{\mathrm{t}}^{2}+2 \mathrm{~m}_{\mathrm{c} \omega} \mathrm{l}_{\mathrm{c} \omega}+\mathrm{J}_{\mathrm{x}}$

$\mathrm{J}=\mathrm{m}_{\mathrm{m}} \mathrm{l}_{\mathrm{m}}^{2}+\mathrm{m}_{\mathrm{t}} \mathrm{l}_{\mathrm{t}}^{2}-2 \mathrm{~m}_{\mathrm{c} \omega} \mathrm{l}_{\mathrm{c} \omega}$,

$\mathrm{K}_{\mathrm{g}}=\left(\mathrm{m}_{\mathrm{m}} \mathrm{l}_{\mathrm{m}}-\mathrm{m}_{\mathrm{t}} \mathrm{l}_{\mathrm{t}}\right) \cos (\theta)+2 \mathrm{~m}_{\mathrm{c} \omega} \mathrm{l}_{\mathrm{c} \omega} \sin (\theta)$

For the detailed derivation of the nonlinear model, the reader is referred to [15]. The parameters of the TRAS model used in this paper are given in Table 1.

The nonlinear model represented in Equation (1) is linearized using Jacobian linearization around the operating points $\theta=\theta_{0}=0[\mathrm{rad}]$ and $\psi=\psi_{0}=$ 
$0[\mathrm{rad}] . \quad$ The state-space representation of the linearized model is represented by the Equation (3).

\begin{tabular}{|c|c|c|c|}
\hline \multicolumn{4}{|c|}{ Table 1: Parameters of the TRAS } \\
\hline Parameter & Value & Parameter & Value \\
\hline $\mathrm{l}_{\mathrm{m}}(\mathrm{m})$ & 0.202 & $\mathrm{~m}_{\mathrm{m}}(\mathrm{Kg})$ & 0.054 \\
\hline $\mathrm{l}_{\mathrm{t}}(\mathrm{m})$ & 0.216 & $\mathrm{~m}_{\mathrm{t}}(\mathrm{Kg})$ & 0.058 \\
\hline $\mathrm{l}_{\mathrm{c} \omega}(\mathrm{m})$ & 0.13 & $\mathrm{~m}_{\mathrm{c} \omega}(\mathrm{Kg})$ & 0.068 \\
\hline $\mathrm{J}_{\mathrm{x}}\left(\mathrm{Kg} / \mathrm{m}^{2}\right)$ & 0.068 & $\mathrm{~g}\left(\mathrm{~m} / \mathrm{s}^{2}\right)$ & 9.8 \\
\hline $\mathrm{j}_{\mathrm{z}}\left(\mathrm{Kg} / \mathrm{m}^{2}\right)$ & 0.002 & - & - \\
\hline
\end{tabular}

$\left[\begin{array}{c}\dot{\psi} \\ \ddot{\psi} \\ \dot{\theta} \\ \ddot{\theta}\end{array}\right]=\left[\begin{array}{cccc}0 & 1 & 0 & 0 \\ 0 & -0.2196 & 0 & 0 \\ 0 & 0 & 0 & 1 \\ 0 & 0 & -1.6685 & -0.4233\end{array}\right]\left[\begin{array}{c}\psi \\ \dot{\psi} \\ \theta \\ \dot{\theta}\end{array}\right]+$ $\left[\begin{array}{lr}0 & 0 \\ 8.05 & 0 \\ 0 & 0 \\ 0 & 6.73\end{array}\right]\left[\begin{array}{l}\mathrm{u}_{\psi} \\ \mathrm{u}_{\theta}\end{array}\right]$

$\left[\begin{array}{l}\psi \\ \theta\end{array}\right]=\left[\begin{array}{llll}1 & 0 & 0 & 0 \\ 0 & 0 & 1 & 0\end{array}\right]\left[\begin{array}{c}\psi \\ \dot{\psi} \\ \theta \\ \dot{\theta}\end{array}\right]+\left[\begin{array}{ll}0 & 0 \\ 0 & 0\end{array}\right]\left[\begin{array}{l}\mathrm{u}_{\psi} \\ \mathrm{u}_{\theta}\end{array}\right]$

here $\psi, \theta$ represent pitch and azimuth angles while $\dot{\psi}$ and $\dot{\theta}$ represent their angular velocities.

One can obtain the transfer function matrix of TRAS from Equation (2) by using $G(s)=C(s I-A)^{-1} B+$ $D$, where $I$ is the identity matrix having same size as $A$ matrix [8] while $\mathrm{A}, \mathrm{B}, \mathrm{C}$ and $\mathrm{D}$ are the state-space matrices. The transfer function matrix of the TRAS can be written as:

$G(s)=\left[\begin{array}{cc}\frac{\psi(s)}{u_{\psi}(s)} & \frac{\psi(s)}{u_{\theta}(s)} \\ \frac{\theta(s)}{u_{\psi}(s)} & \frac{\theta(s)}{u_{\theta}(s)}\end{array}\right]=$

$\left[\begin{array}{cc}\frac{8.05}{s^{2}+0.2191 s} & 0 \\ 0 & \frac{6.73}{s^{2}+0.4233 s+1.669}\end{array}\right]$

In next section, ILC based hybrid control approach is proposed using the above model.

\section{HYBRID ILC BASED CONTROL DESIGN APPROACH}

The TRAS is inherently an unstable system whereas the ILC can ensure the reference tracking for stable systems [28]. It is therefore not possible for ILC to be used directly on the system. Thus, an $\mathrm{H}_{\infty}$ controller has been designed using the linear model to stabilize the system. This $\mathrm{H}_{\infty}$ controller works in the inner loop of the control scheme. Then the ILC has been applied in feedforward path to achieve the lesser control effort while maintaining the tracking performance. $\mathrm{H}_{\infty}$ and ILC approaches are briefly overviewed in the next section.

\section{1 $\mathrm{H}_{\infty}$ Controller}

The $\mathrm{H}_{\infty}$ optimal control problem concerns with finding a controller K(s) that stabilizes system P(s) [29]:

$\left\|\mathrm{F}_{1}(\mathrm{P}(\mathrm{s}), \mathrm{K}(\mathrm{s}))\right\|_{\infty}=\max _{\mathrm{w}} \bar{\sigma}\left(\mathrm{F}_{1}(\mathrm{P}(\mathrm{s}), \mathrm{K}(\mathrm{s}))\right)$

where, $F_{1}(P(s), K(s))$ denotes the lower LFT. The $\mathrm{H}_{\infty}$ norm has several interpretations in terms of performance. One is that it minimizes the peak of the maximum singular value of $\mathrm{F}_{\mathrm{l}}(\mathrm{P}(\mathrm{s}), \mathrm{K}(\mathrm{s}))$ [8]. From here onwards, $\mathrm{P}(\mathrm{s}), \mathrm{K}(\mathrm{s})$ will be denoted by $\mathrm{P}$ and $\mathrm{K}$ respectively. The LFT of $\mathrm{P}$ and $\mathrm{K}$ is given as:

$\mathrm{z}=\mathrm{F}_{1}(\mathrm{P}, \mathrm{K}) \mathrm{w}$

$\mathrm{F}_{1}(\mathrm{P}, \mathrm{K})=\mathrm{P}_{11}+\mathrm{P}_{12} \mathrm{~K}\left(1-\mathrm{P}_{22} \mathrm{~K}\right)^{-1} \mathrm{P}_{21}$

and $\mathrm{P}$ is what is called generalized plant. This interconnection is shown in Fig. 2 where the relationship between the signals can be described by Equations (7-9).

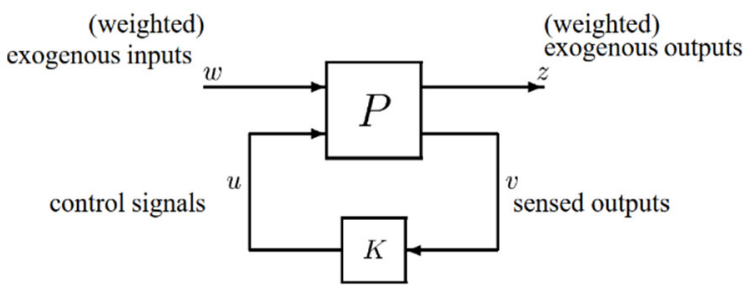

Fig. 2: Generalized Plant Configuration

Objective is to minimize the norm of transfer function from $\mathrm{w}$ to $\mathrm{z}$ and the design problem is to find controller gain $\mathrm{K}$ based on $\mathrm{v}$ which gives $\mathrm{u}$ as control signal to the plant which minimize the closed-loop norm from 
$\mathrm{w}$ to $\mathrm{z}$. The generalized configuration will then be represented as [8]:

$\left[\begin{array}{l}\mathrm{Z} \\ \mathrm{v}\end{array}\right]=\mathrm{P}(\mathrm{s})\left[\begin{array}{l}\mathrm{w} \\ \mathrm{u}\end{array}\right]=\left[\begin{array}{ll}\mathrm{P}_{11}(\mathrm{~s}) & \mathrm{P}_{12}(\mathrm{~s}) \\ \mathrm{P}_{21}(\mathrm{~s}) & \mathrm{P}_{22}(\mathrm{~s})\end{array}\right]\left[\begin{array}{l}\mathrm{w} \\ \mathrm{u}\end{array}\right]$

$\mathrm{u}=\mathrm{K}(\mathrm{s}) \mathrm{v}$

In practice, it is usually not necessary to obtain an optimal controller for the $\mathrm{H}_{\infty}$ problem, and it is often computationally (and theoretically) simpler to design a suboptimal one (i.e. one close to the optimal ones in the sense of the $\mathrm{H}_{\infty}$ norm). Let $\gamma_{\min }$ be the minimum value of $\left\|\mathrm{F}_{1}(\mathrm{P}, \mathrm{K})\right\|_{\infty}$ over all stabilizing controllers $\mathrm{K}$. Then the $\mathrm{H}_{\infty}$ sub-optimal control problem is: given a $\gamma>\gamma_{\text {min }}$, find all stabilizing controllers $\mathrm{K}$ such that:

$\left\|\mathrm{F}_{1}(\mathrm{P}, \mathrm{K})\right\|_{\infty}<\gamma$

To find the $\mathrm{H}_{\infty}$ controller, one has to solve the following optimization problem [29]:

$\begin{array}{ll} & \min _{\gamma, \mathrm{K}}\left\|\mathrm{T}_{\mathrm{z} \rightarrow \mathrm{W}}(\mathrm{P}, \mathrm{K})\right\|_{\infty} \\ \text { such that: } & \gamma>0\end{array}$

K stabilizes P internally and

where $\mathrm{K} \in \mathrm{k}$ and $\mathrm{k}$ is the controller space. The objective function given above is the $\mathrm{H}_{\infty}$ norm of $T_{z \rightarrow w}(P, K)$. Here, $T_{z \rightarrow w}(P, K)$ represents the closedloop system in the lower LFT form introduced above. Thus, the problem is that of $\mathrm{H}_{\infty}$ norm minimization of close-loop performance of the introduced system.

Once a $\mathrm{K}$ is found, it ensures that the $\mathrm{H}_{\infty}$ norm of closed loop system remains less than $\gamma$.

\subsection{Iterative Learning Controller}

ILC uses the information from the past iteration and reduces the error in the next iteration. The control input from the previous iteration is saved in a memory and with some function on error, it is added to the previous control signal to achieve reference tracking. Proportional, integral and derivative control laws can be used to create a new control input based on the current iteration [31].
In order to improve the learning in next iteration, following assumptions are made while designing the ILC.

(1) The starting point of every iteration will always remain the same i.e. if system starting at $\mathrm{t}=0$ has a magnitude of zero as an initial condition, then all the iterations must have the same initial condition

(2) The error after every iteration is converged, that is, the error of second trial must be less than the error of first trial and so on

(3) The time for each iteration is the same

Fig. 3 shows the basic structure of ILC in which $\mathrm{u}(\mathrm{i}, \mathrm{k})$ is the current control input, $y(\mathrm{i}, \mathrm{k})$ is the current output and $\mathrm{u}(\mathrm{i}, \mathrm{k}+1)$ is the control input generated by ILC in the next iteration. The $(\mathrm{i}, \mathrm{k})$ represents the $\mathrm{i}^{\text {th }}$ time interval and $\mathrm{k}^{\text {th }}$ batch or iteration. $\mathrm{u}(\mathrm{i}, \mathrm{k})$ is given to the system and required output response of the system is obtained. All these values are stored in a memory and a new control input is generated in the next iteration so that the desired signal $\mathrm{y}^{\mathrm{d}}(\mathrm{i})$ is perfectly tracked [32].

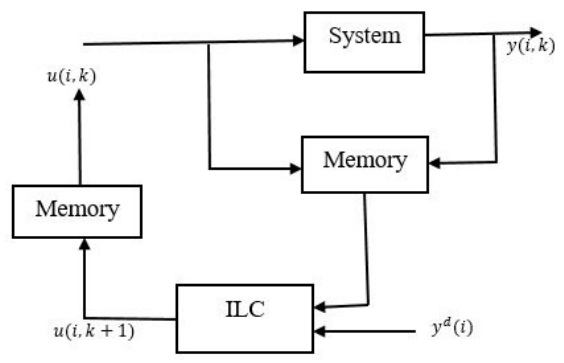

Fig. 3: Basic Structure of ILC

The use of PID controller in a wide range of applications and its effective results motivate to update the ILC law using PID control law. This is known as the PID-type ILC and its mathematical representation is given in the Equation (11) [32]:

$\mathrm{u}(\mathrm{i}, \mathrm{k}+1)=\mathrm{u}(\mathrm{i}, \mathrm{k})+\mathrm{k}_{\mathrm{p}} \mathrm{e}(\mathrm{i}, \mathrm{k})+\mathrm{k}_{\mathrm{d}} \dot{\mathrm{e}}(\mathrm{i}, \mathrm{k})+$ $\mathrm{k}_{\mathrm{i}} \int \mathrm{e}(\mathrm{i}, \mathrm{k}) \mathrm{dt}$

here $\mathrm{k}_{\mathrm{p}}, \mathrm{k}_{\mathrm{d}}, \mathrm{k}_{\mathrm{i}}$ represent the proportional, derivative and integral gains respectively. 
Initial learning gains of PID-type ILC have been obtained by designing the separate PID controllers for each transfer function given by transfer function matrix in Equation (3). Zeigler Nichols PID tuning method is adopted to design the gains of PID controller.

There are many approaches presented by ZieglerNichols for finding gain values, but this work uses traditional technique to tune the PID gains. The PID controller which gives desired output of the closedloop system with unit step input, is mostly tuned with what is called traditional method. The traditional way of tuning the PID parameters uses two constants during the tuning procedure: the time constant $(\mathrm{T})$ and time delay (L) [33]. The curve at the output of the system when the step input is given to it, is known as reaction curve of the system. On the reaction curve, inflection point is pointed out and tangent lines are drawn on the curve at that point. The intersection of this tangent and steady state line gives these constants on $\mathrm{x}$-axis. The system model can then be given by [33]:

$\mathrm{G}(\mathrm{s})=\frac{\mathrm{Ke}}{\mathrm{Ts}+1}$

After acquiring the parameters, the time constants are adjusted in the formula given in Table 2 [33].

\begin{tabular}{|l|c|c|c|}
\hline \multicolumn{4}{|c|}{ Table 2: Zeigler Nichols Table } \\
\hline Controller & $\mathrm{K}_{\mathrm{p}}$ & $\mathrm{T}_{\mathrm{i}}=\frac{1}{\mathrm{~K}_{i}}$ & $\mathrm{~T}_{\mathrm{d}}=\mathrm{K}_{\mathrm{d}}$ \\
\hline PID & $\frac{1.2 \mathrm{~T}}{\mathrm{~L}}$ & $2 \mathrm{~L}$ & $0.5 \mathrm{~L}$ \\
& & & \\
\hline
\end{tabular}

By using the method described above, gains of the PID controller calculated for feedforward PID-type ILC designed in this work, are given as follows:

$\mathrm{k}_{\mathrm{p}}=\left[\begin{array}{cc}1.1 & 0 \\ 0 & 2.1\end{array}\right], \mathrm{k}_{\mathrm{d}}=\left[\begin{array}{cc}0.4 & 0 \\ 0 & 0.2\end{array}\right]$ and

$\mathrm{k}_{\mathrm{i}}=\left[\begin{array}{cc}0.025 & 0 \\ 0 & 0.09\end{array}\right]$

Since the ILC reduces the tracking error in each iteration, it is a necessary condition for the system to be asymptotically stable [28]. TRAS however, possesses the unstable dynamics. To deal with the problem of stability, a hybrid approach is proposed in this paper. $\mathrm{H}_{\infty}$ controller is used in this work to ensure the stability of the TRAS and then ILC is applied in feedforward to reduce the tracking error and minimize the control effort. The block diagram of overall closedloop system is shown in Fig. 4.

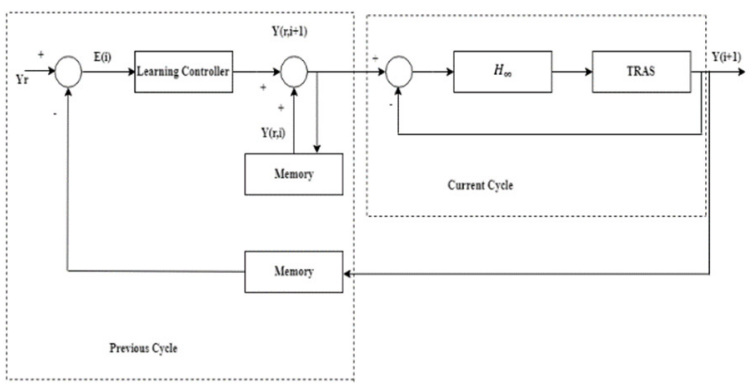

Fig. 4: Closed-Loop Diagram of the Hybrid ILC

Here ILC is used as an external controller without disturbing the existing control system. ILC uses the desired signal and the error stored from the previous cycle to make a new reference trajectory for the existing control system.

\section{RESULTS AND DISCUSSION}

This section shows results of applying controller, obtained through the proposed hybrid ILC technique, on TRAS. It should be emphasized that the plant under consideration is multi-input multi-output and the simulation results have been obtained by applying the proposed controller on the nonlinear model. The results of the strategy are compared with the representative control from approaches already available in literature. For that matter, three control techniques namely, flatness-based control, backstepping control and $\mathrm{H}_{\infty}$ control are chosen for comparison. The first two are nonlinear control techniques and the last one belongs to the class of linear control schemes.

\subsection{Reference Inputs}

Reference inputs are chosen such as to control the movements of TRAS in both horizontal (azimuth angle) and vertical (pitch angle) directions. At first, azimuth angle is increased while keeping the pitch 
angle zero, moving TRAS horizontally in left direction and then pitch angle is also increased resulting in the movement of TRAS both in the horizontal and vertical directions. At the same time, beam of the TRAS is moved in backward direction (azimuth angle is decreased) while keeping it at maximum upward position $(\psi=1.5 \mathrm{rad})$ and vise-versa. By using these reference inputs, one can control the movement of the TRAS in both horizontal and vertical directions. Similar reference inputs, except with smooth edges, are used in [15] for trajectory tracking of TRAS. Sharp reference inputs are very important to ensure the trajectory tracking of TRAS for sudden movement in horizontal and vertical directions, while the smooth reference inputs are applied for slow movements. Both cases are addressed in this paper.

\subsection{Smooth Reference Inputs}

First, the MIMO plant is subjected to smooth reference inputs as shown in Figs. 5-6. It can be seen that all the three control techniques i.e., FBC (Flatness-Based Control), BSC (Back Stepping Control) and $\mathrm{H}_{\infty}$ control as well as the proposed technique work quite well. References are tracked almost perfectly. Plots for both the outputs, that is $\psi$ and $\theta$ and are shown. However, by looking at Fig. 6 that shows control efforts for both the inputs, it becomes apparent that after some iterations the proposed hybrid ILC technique surpasses others. The three techniques being compared; all give control efforts that are higher than the hybrid-ILC approach. In addition, BSC controller has chattering, and therefore it may not be a suitable option for long term use on the plant.

The reference tracking and control efforts (control inputs) with smooth input trajectories at different iterations are shown in Figs. 7-8. It can be seen that the outputs are approaching to the desired output in each next iteration, reducing the tracking error. Similarly, as the iteration number increases, the control effort is reduced. It can also be seen from Fig. 8 that the control effort for $1^{\text {st }}$ iteration is higher than the effort in $5^{\text {th }}$ iteration which is higher than in the $10^{\text {th }}$ iteration. That is, the control effort is reducing in each iteration. By using the hybrid-ILC, not only the reference tracking is improved but this also yields control effort that reduces in each succeeding iteration.
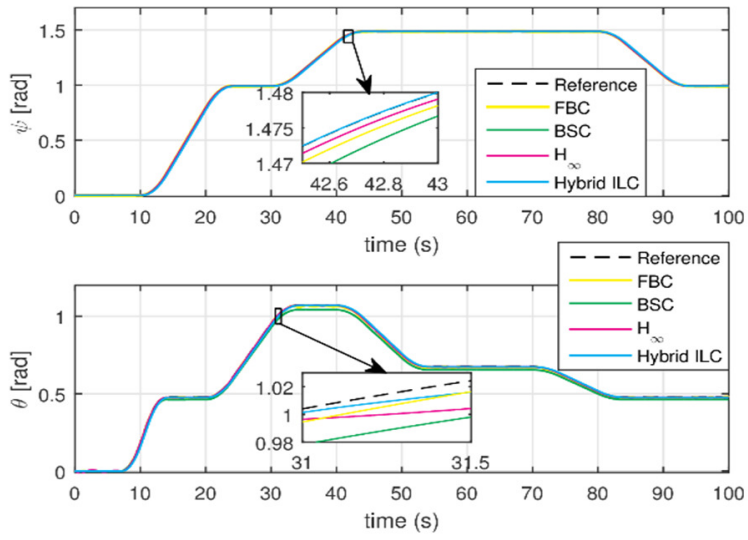

Fig. 5: Comparison of Reference Tracking for Different Controllers with the Hybrid ILC
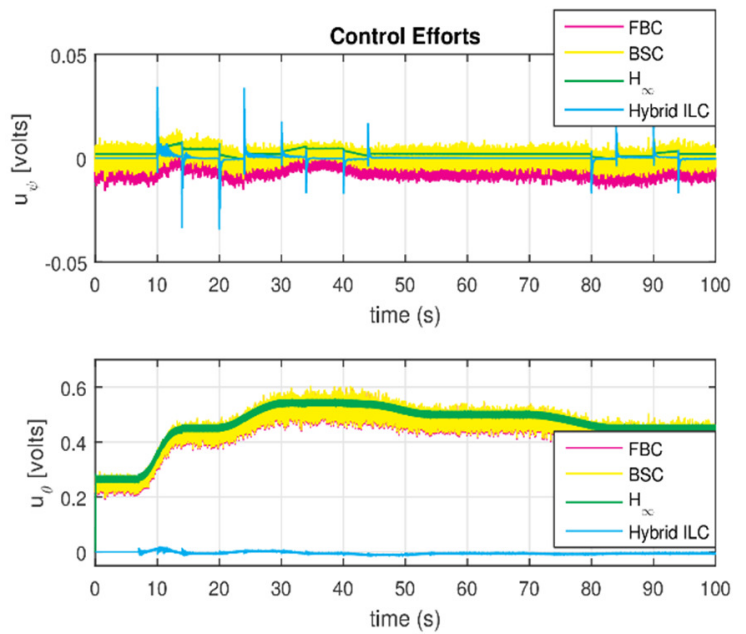

Fig. 6: Comparison of Control Efforts Using Different Controllers with the Hybrid ILC
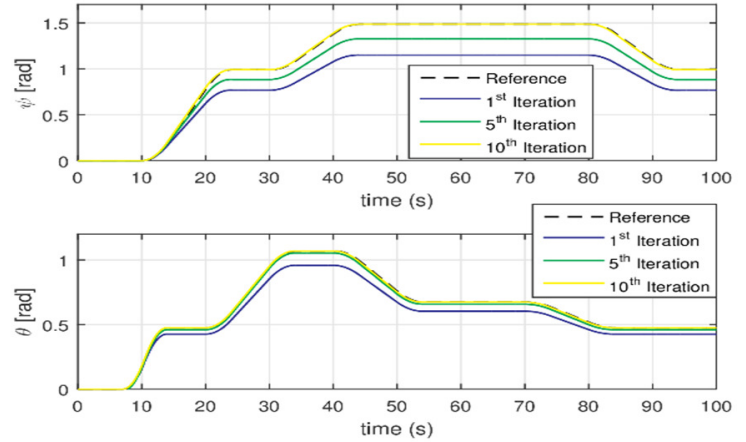

Fig. 7: Reference Tracking at Different Iterations Using Hybrid ILC 

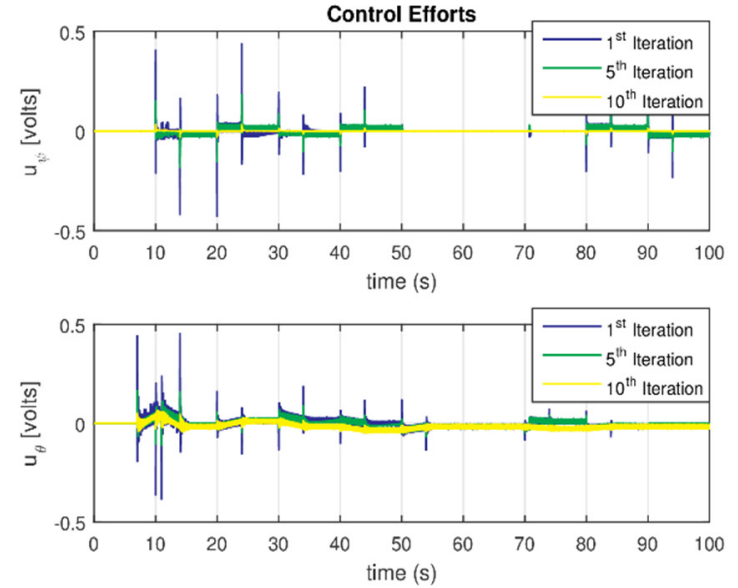

Fig. 8: Control Efforts at different Iterations using Hybrid ILC

\subsection{Sharp Reference Inputs}

Only $\mathrm{H}_{\infty}$ controller is compared with the proposed technique in the sharp reference input case. Controllers obtained through flatness-based approach and the backstepping technique fail to stabilize the system in closed-loop when subjected to sharp reference inputs. Though the same controllers were able to stabilize and track the references in the smooth case, in the sharp case these are clearly insufficient. The flatness-based and backstepping control laws require $1^{\text {st }}$ and $2^{\text {nd }}$ derivatives of the reference trajectory. But in case of the reference inputs with sharp edges, the derivatives become very high leading to the instability of the closed-loop system.

The $\mathrm{H}_{\infty}$ controller is able to track the reference, as shown in Fig. 9, and therefore is considered in this section. Again, both the $\mathrm{H}_{\infty}$ controller and the proposed hybrid-ILC both track the reference well. However, hybrid-ILC controller achieves this after a few iterations, which in this case is 8 . But the control effort required for trajectory tracking is lesser in case of hybrid-ILC. Both the outputs, that is $\psi$ and $\theta$ are tracked well after 8 iterations. Fig 10 shows the control effort for the sharp reference case. It can be seen from the figure that the control effort required to track the reference trajectory with sharp edges is smaller than that for the case of $\mathrm{H}_{\infty}$.
At the outset it may appear that the learning of controller gains may take a longer process, and, in some instances indeed this might be the case. However, the advantage of control effort reduction has a direct bearing on the input energy. In both smooth and sharp reference cases, the energy of the input signals to the plant generated by the controller is less than all the other three controllers. This is especially significant for $\mathrm{u}_{\theta}$ input, i.e. 11. In the sharp edges case for example it is 225.29 for $\mathrm{H}_{\infty}$ controller and 1.10 for the proposed controller which is more than a 200 times improvement. Table 3 shows the comparison of the 2 norm of the control efforts of the proposed approach with that of flatness-based, backstepping and $\mathrm{H}_{\infty}$ controllers for both cases, i.e. with the smooth and sharp reference trajectories. The values are calculated by taking 2-norms of the corresponding input signals. Another long-term effect on the actuator is that it is not pushed to the limits and thus experiences less wear and tear.
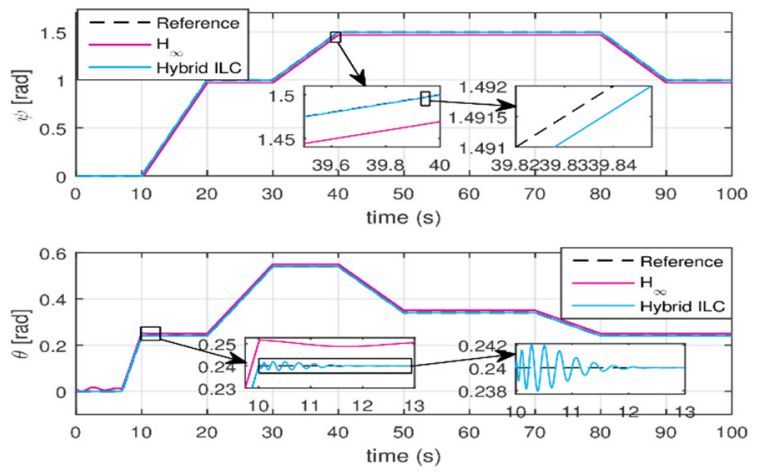

Fig. 9: Reference Tracking with Sharp Edges
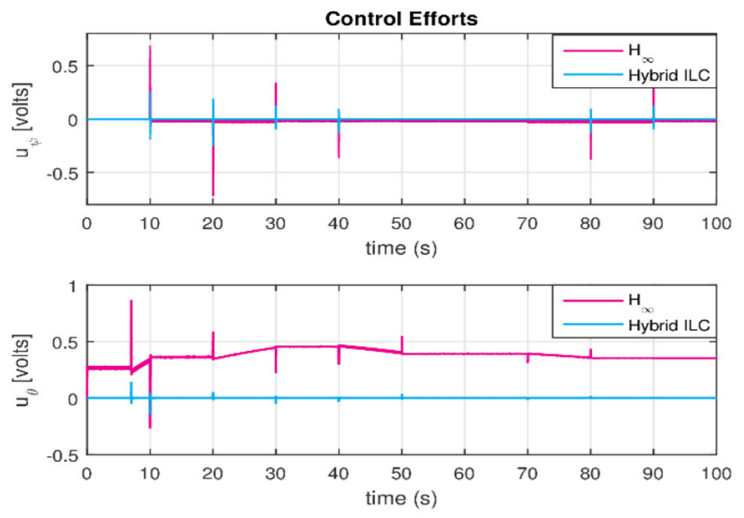

Fig. 10: Control Effort for Reference Input with Sharp Edges 


\begin{tabular}{|c|c|c|c|c|}
\hline \multicolumn{5}{|c|}{ Table 3: Comparison of $\mathrm{L}_{2}$ Norm of Control } \\
Efforts \\
\hline \multirow{3}{*}{ Controller } & \multicolumn{5}{|c|}{ Control Efforts } \\
\cline { 2 - 5 } & \multicolumn{2}{|c|}{ Smooth Edges } & \multicolumn{2}{c|}{ Sharp Edges } \\
\cline { 2 - 5 } & $\left\|\mathrm{u}_{\psi}\right\|_{2}$ & $\left\|\mathrm{u}_{\theta}\right\|_{2}$ & $\left\|\mathrm{u}_{\psi}\right\|_{2}$ & $\left\|\mathrm{u}_{\theta}\right\|_{2}$ \\
\hline $\begin{array}{c}\text { Flatness } \\
\text { based }\end{array}$ & 0.85 & 101.89 & --- & --- \\
\hline Backstepping & 0.94 & 270.38 & --- & --- \\
\hline $\mathrm{H}_{\infty}$ & 0.64 & 101.24 & 10.82 & 225.29 \\
\hline Hybrid ILC & 0.35 & 11 & 2.95 & 1.10 \\
\hline
\end{tabular}

The reference tracking and control efforts (control inputs) for input trajectories with sharp edges at different iterations are shown in Figs. 11-12. The control effort required for tracking in the next iteration is smaller than that in the previous iteration.
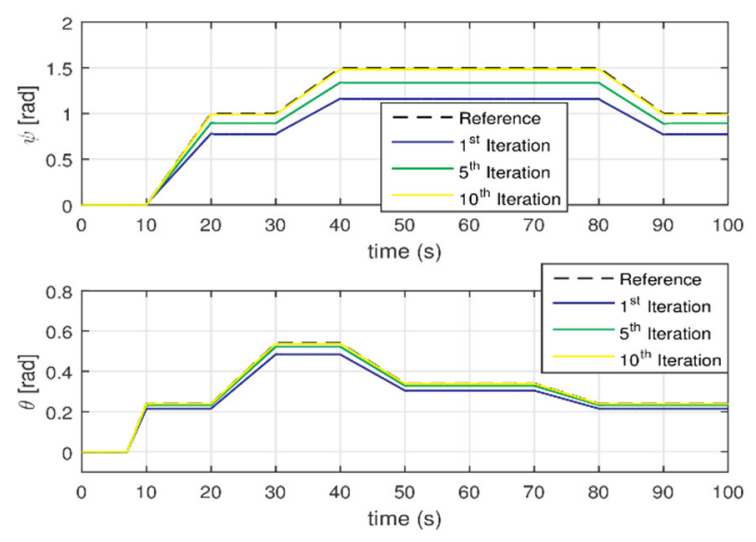

Fig. 11: Reference Tracking at Different Iteration of Hybrid ILC for Reference Trajectory with Sharp Edges
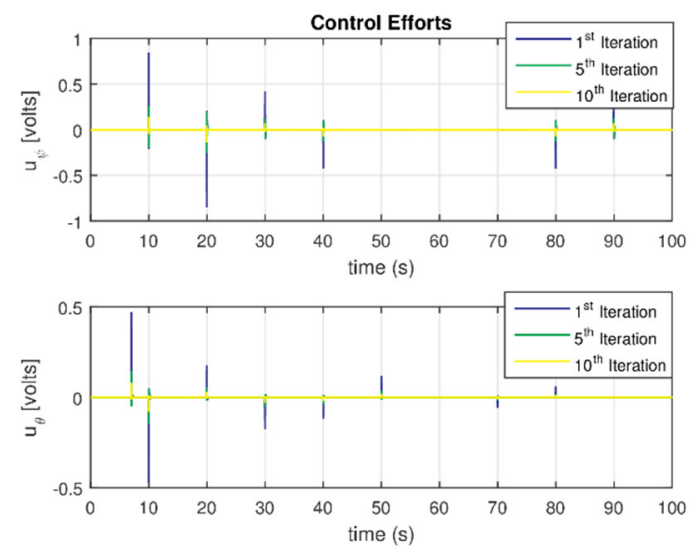

Fig. 12: Control effort at Different Iteration of Hybrid ILC for Reference Trajectory with Sharp Edges
The tracking error of output for the hybrid-ILC at different iterations is shown in Fig. 13. It can be clearly seen that the error decreases monotonically in each iteration and finally becomes zero at the $8^{\text {th }}$ iteration. A similar approach with Fractional Order PID controller in inner loop is proposed in [27] for the SISO Magnetic Levitation System, which is able to achieve the reference tracking with $1 \%$ tracking error, after 70 iterations. However, the improved hybrid-ILC presented in this paper is able to achieve zero tracking error after 8 iterations showing the effectiveness of the proposed approach.

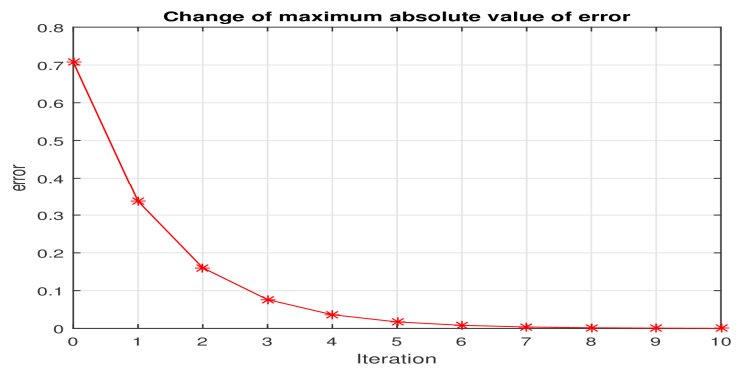

Fig. 13: Tracking Error at Different Iterations using Hybrid-ILC

\section{CONCLUSION}

This paper achieves the objective of trajectory tracking of sharp references for the Twin Rotor Aerodynamic System using a hybrid-ILC approach. The system is stabilized using an $\mathrm{H}_{\infty}$ controller in the inner loop and then a PID-type ILC is designed to minimize the tracking error. The error is monotonically decreasing in each iteration and becomes zero in $8^{\text {th }}$ iteration.

The comparison of proposed approach with the FBC and BSC shows that the proposed approach has the reduced control effort. The FBC and BSC laws require $1^{\text {st }}$ and $2^{\text {nd }}$ derivatives of the reference trajectory. But in case of the reference inputs with sharp edges, the derivatives become very high leading to the instability of closed-loop system. The proposed controller successfully tracks the reference trajectory.

\section{ACKNOWLEDGEMENTS}

The authors would like to acknowledge the University of Engineering \& Technology, Taxila, Pakistan, for Mehran University Research Journal of Engineering and Technology, Vol. 40, No. 1, January 2021 [p-ISSN: 0254-7821, e-ISSN: 2413-7219] 
providing all the facilities required to complete the research. The authors also like to acknowledge the comments made by anonymous reviewers.

\section{REFERENCES}

[1] Valavanis K.-P., Vachtsevanos G.-J., "Handbook of Unmanned Aerial Vehicles", Springer, 2015.

[2] "Two Rotor Aero-Dynamical System User's Manual”, INTECO, 2013.

[3] Venkatesan C., "Fundamentals of Helicopter Dynamics", CRC Press, 2014.

[4] Juang J.-G., Huang M.-T., W.-K. Liu, "PID control using presearched genetic algorithms for a MIMO system", IEEE Transactions on Systems Man and Cybernetics, Part C (Applications Rev., Vol. 38, No. 5, pp. 716727, 2008.

[5] Khizer A.-N., Soomro A.-M., Ali, S.-A., "A Hybrid Flight Control for a Simulated Raptor-30 V2 Helicopter", Mehran University Research Journal of Engineering and Technology, Vol. 34, No. 2, pp. 176-182, 2015.

[6] Aldbrez F.-M., Alam M.-S., Tokhi, M.-O., "Input-shaping with GA-tuned PID for target tracking and vibration reduction", Proceedings of the IEEE Mediterrean International Symposium on Intelligen Control and Automation, pp. 485-490, 2005.

[7] Wen P., Lu, T.-W., "Decoupling control of a twin rotor MIMO system using robust deadbeat control technique", IET Control Theory and Applications, Vol. 2, No. 11, pp. 999-1007, 2008.

[8] Skogestad S., Postlethwaite, I., "Multivariable Feedback Control: Analysis and Design", Wiley New York, Vol. 2, 2007.

[9] Pratap B., Agrawal A., and Purwar S., "Optimal control of twin rotor MIMO system using output feedback", Proceedings of the 2nd International Conference on Power, Control and Embedded Systems (ICPCES), , 2012, pp. 1-6, 2012.

[10] Phillips A., Sahin F., "Optimal control of a twin rotor MIMO system using LQR with integral action", Proceedings of the World Automation Congress (WAC), pp. 114-119, 2014.

[11] John L., Mija S. J., "Robust Hळ control algorithm for Twin Rotor MIMO system", Proceedings of the IEEE International Conference on Advanced Communications, Control and Computing Technologies, pp. 168-173, 2014.

[12] Ahmad S.-M., Chipperfield A.-J. Tokhi O., "Dynamic modeling and optimal control of a twin rotor MIMO system", Proceedings of the IEEE National Aerospace and Electronics Conference, pp. 391-398, 2000.

[13] Rahideh A., Shaheed M.-H., "Stable model predictive control for a nonlinear system", Journal of the Franklin Institute, Vol. 348, No. 8, pp. 1983-2004, 2011.

[14] Butt S.-S., Sun H., Aschemann H., "Comparison of Backstepping-Based Sliding Mode and Adaptive Backstepping for a Robust Control of a Twin Rotor Helicopter", Variable-Structure Approaches, Springer, pp. 3-30, 2016.

[15] Sun H., Butt S.-S., Aschemann H., "Discretetime flatness-based control for a twin rotor helicopter with an Extended Kalman filter", Proceedings of the IEEE/ASME International Conference on Advanced Intelligent Mechatronics, AIM, 2016.

[16] Martinez M.-L, Diaz J.-M., Ortega M.-G., and Rubio, F.-R., "Control of a laboratory helicopter using switched 2-step feedback linearization", Proceedings of the American Control Conference, Vol. 5, pp. 4330-4335, 2004.

[17] Rotondo D., Nejjari F., Puig V., "Quasi-LPV modeling, identification and control of a twin rotor MIMO system," Control Engineering Practice, Vol. 21, No. 6, pp. 829-846, 2013.

[18] Zeghlache S., Bouguerra A., Ladjal M., "Sliding mode controller using nonlinear sliding surface applied to the 2-DOF helicopter", Proceedings of the International Conference on Electrical and Information 
Technologies (ICEIT), 2016, pp. 332-337, 2016.

[19] Tao C.-W., Taur J.-S., Chang Y.-H., Chang C.-W., "A novel fuzzy-sliding and fuzzyintegral-sliding controller for the twin-rotor multi-input--multi-output system", IEEE Transactions on Fuzzy Systems, Vol. 18, No. 5, pp. 893-905, 2010.

[20] Bouarroudj N., Djari A., Boukhetala, D., Boudjema F., "Tuning of decentralized fuzzy logic sliding mode controller using PSO algorithm for nonlinear Twin Rotor Mimo System", Proceedings of the 6th International Conference on Systems and Control (ICSC), pp. 45-50, 2017.

[21] Mondal S., Mahanta C., "Second order sliding mode controller for twin rotor MIMO system", Proceedings of the Annual IEEE India Conference (INDICON), pp. 1-5, 2011.

[22] Mondal S. Mahanta C., "Adaptive secondorder sliding mode controller for a twin rotor multi-input--multi-output system", IET Control Theory and Applications, Vol. 6, No. 14, pp. 2157-2167, 2012.

[23] Rashad R., Aboudonia A., Badawy A.-E, “A novel disturbance observer-based backstepping controller with command filtered compensation for a MIMO system", Journal of the Franklin Institute, Vol. 353, No. 16, pp. 4039-4061, 2016.

[24] Haruna A., Mohamed Z., Efe M.-Ö., Basri M.-M., "Dual boundary conditional integral backstepping control of a twin rotor MIMO system", Journal of the Franklin Institute, Vol. 354, No. 15, pp. 6831-6854, 2017.

[25] Butt S.-S., Prabel R., Aschemann H., "Multivariable flatness-based control of a helicopter with two degrees of freedom," Control, Decision and Information Technologies (CoDIT), pp. 321-326, 2014.

[26] Sun H., Butt S., Aschemann H., "Discretetime flatness-based control for a twin rotor helicopter with an Extended Kalman filter", Proceedings of the IEEE International Conference on Advanced Intelligent Mechatronics (AIM), pp. 507-512, 2016.
[27] Hanif B., "Iterative Learning Control based Fractional Order PID Controller for Magnetic Levitation System", University of Engineering and Technology, MSc Thesis, 2018.

[28] Shen D., "Iterative learning control with incomplete information: a survey", IEEE/CAA Journal of Automatica Sinica, Vol. 5, No. 5, pp. 885-901, 2018.

[29] Wang S.-M., Fang M.-C., Hwang C.-N., "Vertical Obstacle Avoidance and Navigation of Autonomous Underwater Vehicles with $\mathrm{H} \infty$ Controller and the Artificial Potential Field Method", Journal of Navigation, Vol. 72, No. 1, pp. 207-228, 2019.

[30] Apkarian P., Noll D., "The $\mathrm{H} \infty$ control problem is solved," AerospaceLab J., No. 13, 2017.

[31] Ketelhut M., Stemmler S., Gesenhues J., Hein M., Abel D., "Iterative learning control of ventricular assist devices with variable cycle durations", Control Engineering. Practice, Vol. 83, pp. 33-44, 2019.

[32] Xu J.-X., "A survey on iterative learning control for nonlinear systems", International Journal of Control, Vol. 84, No. 7, pp. 12751294, 2011.

[33] Åström K. J., Hägglund T., "PID Controllers: Theory, Design, and Tuning", Instrument society of America Research Triangle Park, NC, 1995. 